Vol. 19, $n^{\circ} 1 \mid 2015$

Varia

\title{
Antoine Follain, Bruno Lemesle, Michel Nassiet, Éric Pierre, Pascale Quincy-Lefebvre (dir.), La violence et le judiciaire : discours, perceptions, pratiques
}

Rennes, Presses Universitaires de Rennes, « Histoire », 2008, 383 pp., ISBN 9782753505599

Ludovic Maugué

\section{(2) OpenEdition}

\section{Journals}

Édition électronique

URL : http://journals.openedition.org/chs/1576

DOI : $10.4000 /$ chs. 1576

ISSN : 1663-4837

Éditeur

Librairie Droz

Édition imprimée

Date de publication : 1 juin 2015

Pagination : 136-138

ISBN : 978-2-600-01953-8

ISSN : $1422-0857$

Référence électronique

Ludovic Mauqué, « Antoine Follain, Bruno Lemesle, Michel Nassiet, Éric Pierre, Pascale Quincy-

Lefebvre (dir.), La violence et le judiciaire : discours, perceptions, pratiques », Crime, Histoire \& Sociétés /

Crime, History \& Societies [En ligne], Vol. 19, n¹ | 2015, mis en ligne le 01 juin 2017, consulté le 24

septembre 2020. URL : http://journals.openedition.org/chs/1576; DOI : https://doi.org/10.4000/chs 1576

Ce document a été généré automatiquement le 24 septembre 2020.

(C) Droz 


\section{Antoine Follain, Bruno Lemesle, Michel Nassiet, Éric Pierre, Pascale Quincy-Lefebvre (dir.), La violence et le judiciaire : discours, perceptions, pratiques}

Rennes, Presses Universitaires de Rennes, « Histoire », 2008, 383 pp., ISBN 9782753505599

\section{Ludovic Maugué}

\section{RÉFÉRENCE}

Antoine Follain, Bruno Lemesle, Michel Nassiet, Éric Pierre, Pascale Quincy-Lefebvre (dir.), La violence et le judiciaire : discours, perceptions, pratiques, Rennes, Presses Universitaires de Rennes, « Histoire », 2008, 383 pp., ISBN

9782753505599.

1 Formant les actes du colloque international réuni à Angers en mai 2006 et conjointement organisé par le Centre d'histoire des régulations sociales (HIRES) et le Centre de recherche historique de l'Ouest (CERHIO), les textes recueillis dans cet ouvrage ont pour objectif commun de considérer la violence dans ses relations avec le judiciaire. Selon les organisateurs, le malaise de la justice - symbolisé alors en France par l'affaire d'Outreau - invite à apprécier «la place occupée par la violence dans le travail judiciaire; une place qui est un indice des différentes fonctions attribuées à la justice à différents moments de notre histoire » (p. 10).

2 En soulignant dans leur solide introduction la polysémie du mot et le caractère extensif et diffus de la notion (en France la violence n'est qualifiée juridiquement qu'en 1863), Bruno Lemesle, Michel Nassiet et Pascale Quincy-Lefebvre posent les jalons d'une 
lecture de la violence sur la longue durée, du haut Moyen Âge à la $\mathrm{V}^{\mathrm{e}}$ République. Afin d'évacuer les perceptions subjectives, qui « rendent improbables la définition d'un objet "violence" [et] entraînent surtout une dilution de sens qui en affaiblit la portée »(p.10), ils signalent l'indispensable attention accordée aux réponses sociales et judiciaires pour appréhender le phénomène. Ainsi, en la reconnaissant, le judiciaire confère-t-il à la violence un statut objectif puisqu'il l'insère dans un système. Le propos de cet ouvrage collectif consiste donc à examiner comment l'institution judiciaire définit et répond à la violence, et dans quelle mesure la justice sécrète elle-même d'autres formes de violence. Pour ce faire, il convient toutefois d'éviter l'écueil d'une analyse de la violence du passé menée à l'aune des conceptions actuelles; en effet, selon le contexte historique, certains types de violence ont pu bénéficier d'une légitimité grâce à une acceptation sociale.

Dès lors, les emplois du terme "violence " font l'objet d'un examen sémantique, qui révèle que si la violence est corrélée à son traitement judiciaire dès le haut Moyen Âge, force est d'en constater l'absence de définition juridique. Au regard des codes de lois des rois barbares (Édit de Rothari, Leges visigothorum), ce vocable concerne fréquemment le viol de la femme mariée, de la jeune fille ou de la servante, mais aussi, par association de mots, la violation des espaces ainsi que la seditio (soulèvement). Le registre s'avère encore plus vaste si l'on considère les textes de l'Église (Décret de Gratien), pour lesquels la notion de violence s'étend à tout ce qui porte atteinte au sacré. Enfin, à partir du $\mathrm{XI}^{\mathrm{e}}$ siècle, les documents issus de la pratique judiciaire attestent d'un usage du terme " violence " pris dans le sens d'acte commis à tort, contre le droit. Au-delà de la traduction de violentia - qu'il faut rendre par «injustice » -, les occurrences du mot "violence» et de ses dérivés désignent toujours des actions injustes : "c'est parce qu'un acte a été accompli sans avoir reçu la validation d'une instance judiciaire qu'il peut être qualifié de violent [...]. Ainsi la violence désigne-t-elle l'injustice avant de désigner l'action physique, la voie de fait au sens actuel » (pp. 13-14).

À la faveur d'un stimulant survol historiographique, l'introduction s'attache ensuite à examiner le renouvellement récent des réflexions sur la violence au Moyen Âge et à l'époque moderne. L'analyse de ces travaux débouche sur la constatation que si le lien entre violence et judiciaire était parfois sous-entendu, voire évoqué, il n'avait encore jamais été approfondi. Les auteurs de l'introduction dégagent alors une grille des questionnements les plus récents qui s'articule autour de trois axes de réflexion : la violence comme stratégie et comme code ; les formes d'acceptation sociale et légale de la violence ; la construction de la violence. Longtemps dominée par la question des processus de régulation des homicides à l'origine de notre modernité aux XIX ${ }^{\mathrm{e}}$ et $\mathrm{XX}^{\mathrm{e}}$ siècles, l'historiographie de la violence pour l'époque contemporaine connaît un renouvellement dans les années 1990 au contact de l'anthropologie et dans la foulée des recherches menées par Michel Foucault. Appréhendée sous des formes nouvelles (violences de guerre, violence extrême, violence médiatisée, travaux sur l'enfance, mais également sur les femmes), marquée par l'émergence de la notion de victime, la violence contemporaine a acquis, selon les auteurs, "une visibilité publique qui la transforme en objet de discours dans une société d'opinion qui [...] s'organise autour de la défense des droits de l'individu » (p. 20). 
5 Proposées selon une approche diachronique, les vingt-quatre contributions retenues par les organisateurs du colloque d'Angers s'attèlent à débusquer les inflexions du diptyque violence-judiciaire, du Moyen Âge à nos jours. Regroupées en quatre parties d'ampleurs inégales ("Violence admise, tolérée, pardonnée »; "violence quotidienne et tribunaux»; «construction de la violence, différences culturelles et géographiques »; "violence et contexte de guerre»), ces communications portent essentiellement sur l'espace politique français, cinq d'entre elles se situant toutefois dans d'autres aires géographiques (villes castillanes, Venise, Québec, Naples et Italie du Sud).

6 S'inscrivant entre le bas Moyen Âge et l'époque moderne, la problématique des normes et des seuils est traitée dans la première partie au travers des "violences admises, tolérées, pardonnées ». Le thème de la violence légale des « humbles » aux $\mathrm{XII}^{\mathrm{e}}$ et début XIII ${ }^{e}$ siècles est ainsi examiné à la lecture des premiers textes normatifs gascons. En révélant qu'il existe en Gascogne un droit à la violence pour les humbles, Hélène Couderc-Barraud précise que, quoique anachronique, le concept de "violence légale » s'avère néanmoins opérant. Sont notamment abordées ensuite la question du pardon des violences dans les lettres de rémission $\mathrm{du} \mathrm{XV}$ siècle (Pierre Charbonnier), et celle de la place du système vindicatoire dans la société d'Ancien Régime. Alors que Michel Nassiet s'emploie à observer l'ensemble des actes de violence considérés comme légitimes dans le cadre des relations de parenté au XVI ${ }^{\mathrm{e}}$ siècle - et appelle, à la suite de Claude Gauvard ${ }^{1}$, à "nuancer considérablement les théories qui construisent la "civilisation des mœurs" sur la sauvagerie initiale d'un temps où aurait régné une violence "pulsionnelle" » (p. 87) -, Stuart Carroll se propose, en prolongement de son livre Blood and violence in early modern France ${ }^{2}$, d'étudier la faide (le duel et la vengeance) en considérant trois éléments constitutifs de la sociabilité de vengeance, soit la construction de la mémoire sociale, le rôle de conseil au sein des familles, et enfin le comportement des vengeurs dans l'espace public.

7 La deuxième partie est consacrée aux violences quotidiennes. Hormis une enquête sur les avatars judiciaires des "violences conjugales" au XIX" siècle (Victoria Vanneau) et une étude du quotidien du tribunal correctionnel de Blois entre 1815 et 1848 (Stéphane Vautier), les contributions qui la composent interrogent le traitement judiciaire réservé aux injures, qu'elles soient commises contre les auxiliaires subalternes des justices seigneuriales angevines entre le bas Moyen Âge et le $\mathrm{XVI}^{\mathrm{e}}$ siècle (Isabelle Mathieu), jugées dans un tribunal de première instance sous l'Ancien Régime (Hervé Piant) ou réparées devant les justices de paix au XIX siècle (Vincent Bernaudeau). Regroupant aussi bien les insultes verbales que les violences physiques légères, sous l'Ancien Régime, l'injure est indissociable de la notion d'honneur, alors au cœur des relations entre les hommes. Aussi la réponse que l'institution judiciaire réserve à ce type d'affaires est-elle révélatrice du rôle endossé par la justice dans le jeu subtil de l'interaction sociale.

8 Attentive à l'impact des différences culturelles et des ancrages géographiques dans la construction des violences, la troisième partie est la plus conséquente de l'ouvrage (10 communications), mais aussi la plus hétéroclite. Citons pêle-mêle certains des thèmes qui la composent : construction de la torture comme violence légale dans le cadre de la chasse aux sorcières suivant l'exemple de la Vauderie d'Arras (Franck Mercier), répression judiciaire des violences militaires sous Louis le Juste (Hélène Fernandez), étude statistique du Compte général de la justice criminelle (Bruno Aubusson de Cavarlay), 
rapports entre violence, hommes et justice au Québec entre 1780 et 1860 (Donald Fyson), analyse de l'organisation de la Camorra napolitaine au XIX ${ }^{e}$ siècle (Marcella Marmo), mais aussi étude comparée des modèles de justice des mineurs entre la France, le Canada et la Belgique au cours de la première moitié du XX siècle (David Niget).

Les trois derniers articles qui forment la quatrième partie de ce livre sont réunis sous le thème "violence et contexte de guerre ». Particulièrement mal connu, le rôle des avocats devant la justice militaire allemande chargée de juger les civils français entre 1940 et 1944 est examiné par Serge Defois. Son attention se porte d'une part sur les conditions d'exercice de la défense devant une juridiction à caractère exceptionnel, et d'autre part sur l'éventualité d'une instrumentalisation des avocats nantais dans l'optique d'une criminalisation de la résistance. En revisitant l'analyse des violences populaires des années 1944-1945 (exécutions, mais également formes de violences physiques, matérielles ou sociales), Marc Bergère sonde leurs modes de régulation et examine le processus de légitimation (ou de «délégitimation») de la violence et des victimes à l'échelle des communautés et des autorités. Enfin, le traitement judiciaire de la violence internationale depuis la fin du monde bipolaire fait l'objet de la contribution d'Yves Denéchère, qui conclut que "sous l'aiguillon de l'opinion publique internationale et des $\mathrm{ONG}$, de nouvelles normes internationales des violences de guerre semblent pouvoir s'imposer » (p. 379).

Enfin, ce livre est conclu par les remarquables mises en perspectives de Xavier Rousseaux, lequel résume les résultats acquis, mais thématise également les difficultés théoriques et méthodologiques posées par le couple violence-judiciaire, en dégageant les ancrages majeurs de la réflexion (émergence tardive d'un vocabulaire de la violence ; transformation de la communauté de face-à-face en État ; difficulté de parler de violence et judiciaire en termes de droit). En restituant habilement l'ouvrage dans l'historiographie de la violence et du judiciaire, Xavier Rousseaux soumet les communications à une quintuple opération, soit: préciser les sources, cerner les représentations, replacer aux contextes de société, saisir des pratiques judiciaires et proposer une interprétation d'ensemble.

\section{NOTES}

1. Claude Gauvard, "De grâce especial ", Crime, État et société en France à la fin du Moyen Âge, Paris, Publications de la Sorbonne, 1991.

2. Stuart Carroll, Blood and violence in early modern France, Oxford, Oxford UP, 2006. 


\section{AUTEURS}

LUDOVIC MAUGUÉ

Université de Genève 